Pacific Journal of Mathematics

THREEFOLDS WHOSE HYPERPLANE SECTIONS ARE 


\section{THREEFOLDS WHOSE HYPERPLANE SECTIONS ARE ELLIPTIC SURFACES}

\section{HARRY D'SOUZA}

In this paper we classify pairs $(X, S)$ where $X$ is a smooth complex projective threefold and $S$ is a smooth ample divisor in $X$. Moreover $S$ is elliptic and $\kappa(S)=1$. We use the logarithmic Kodaira dimension of $(X, S)$ as the basis of classification. Sommese studied such pairs in "The birational theory of hyperplane sections of projective threefolds" where he showed that such pairs $(X, S)$ can be reduced to $\left(X^{\prime}, S^{\prime}\right)$, where $S^{\prime}$ is ample in $X^{\prime}$, and $S^{\prime}$ is minimal model of $S$. In the case when $S$ is elliptic, with $h^{1,0}(S) \neq 0$ he showed that one obtains a surjective morphism $p$, from $X$ onto a smooth curve $Y$ such that this morphism restricted to $S$ is a reduced elliptic fibration. Shepherd-Barron proved the same result using Mori's methods without the restriction on $h^{1,0}(S)$. We state these results in $\S 0$.

We show that the general fibres of $p$ are del Pezzo surfaces and classify these in the case where they are of degrees $1,2,3,4,7,8$ and 9. We show that in the degree 9 case that it is indeed a $\mathbf{P}^{2}$-bundle over $Y$. In the degree $8\left(\cong \mathbf{P}^{1} \times \mathbf{P}^{1}\right)$ case we have a birational morphism to a $\mathbf{P}^{2}$-bundle.

0. Notation and background material. For the most part we will follow the notation in [Ha 1] as closely as possible. For the convenience of the reader we have included as much as possible. In this section we state most of the theorems that have been used.

(0.1) If $\mathscr{S}$ is a sheaf of abelian groups on a topological space $X$, then the global sections of $\mathscr{S}$ over $X$ are denoted by $\Gamma(\mathscr{S})$, or by $\Gamma(X, \mathscr{S})$ in case of ambiguity.

(0.2) All manifolds and spaces are complex analytic, unless specified otherwise. All dimensions are over C. Complex analytic is often abbreviated to analytic. The sheaf of $X$ is denoted by $\mathscr{O}_{X}$. We do not distinguish between a holomorphic vector bundle on $X$, and its sheaf of holomorphic sections. Hence a tensor product of a vector bundle and a coherent analytic sheaf is actually the appropriate sheaves being tensored together over $\mathscr{O}_{X}$.

(0.3) If $\mathscr{S}$ denotes a coherent analytic sheaf on $X$, then $\chi(X, \mathscr{S})$ or $\chi(\mathscr{S})$ denotes its Euler characteristic.

(0.4) When the exact dimension of a projective space is irrelevant, then we denote it by $\mathbf{P}$. 
(0.5) We shall use the words morphism and holomorphic map interchangeably. We denote the restriction of a morphism $p: X \rightarrow Y$, where $Y$ is a complex analytic space by $p_{S}$ or $\left.p\right|_{S}, S$ being an analytic subspace of $X$.

(0.5.1) Definition. If $L$ denotes a line bundle on $X$, a complex projective variety, then $L$ is very ample if $\Gamma(L)$ spans $L$, and the map $\Phi: X \rightarrow \mathbf{P}$ is an embedding.

(0.5.2) A line bundle $L$ on a projective variety $X$ is ample if there is an integer $n$ such that $L^{n}$ is very ample. See [Ha 2] for a detailed account.

(0.5.3) If $S$ is ample in $X$, where $S$ and $X$ are smooth, then the logarithmic Kodaira dimension of $(X, S)$ is defined as follows,

$$
\log (X, S)=\operatorname{tr}_{\mathbf{C}}\left(\bigoplus_{n \geq 0} \Gamma\left(X,\left(K_{X} \otimes S\right)^{n}\right)\right)-1
$$

where $K_{X}$ is the canonical divisor of $X$. For a more general definition see [I1 or I2].

(0.5.4) We denote the $i$ th direct image sheaf by $p_{(i)}$ instead of $R^{i} p_{*}$.

Below we shall state without proof, some of the results on minimal pairs. See [F-So] for details.

(0.6) TheOREM. Let $L$ be an ample line bundle on $X$, a connected smooth projective threefold. Assume that there is a smooth $S$ in $|L|$, of non-negative Kodaira dimension. Assume also that $X$ is not a holomorphic $\mathbf{P}^{1}$-bundle over a smooth surface $\tilde{S}$, with smooth $S$ in $|L|$ as meromorphic sections. Then there exists an ample line bundle $L^{\prime}$ over a projective manifold $X^{\prime}$, such that:

(a) $X$ is the blow-up $\pi: X \rightarrow X^{\prime}$, of $X^{\prime}$ at a finite set $F$ in $X^{\prime}$,

(b) sending smooth $S^{\prime}$ in $\left|L^{\prime}-F\right|$ to their proper transforms on $X$, gives a one-to-one correspondence between smooth $S^{\prime}$ in $\left|L^{\prime}-F\right|$ and smooth $S$ in $|L|$,

(c) all smooth $S$ in $|L|$ are of non-negative Kodaira dimension, and $\pi_{S}: S \rightarrow \pi(S)=S^{\prime}$ maps $S$ onto its minimal model.

(0.6.1) REMARK. This pair $\left(X^{\prime}, L^{\prime}\right)$ is the minimal model associated to $(X, L)$ or $\left(X^{\prime}, S^{\prime}\right)$ is the minimal model associated with $(X, S)$.

The following theorem was proved by Sommese under the condition $h^{1,0}(S) \neq 0$, but using the results of Mori [M], Shepherd-Barron removed the condition. 
(0.7) THEOREM. Let $S$ be a smooth ample divisor on a smooth connected threefold $X$. Assume that $S$ is an elliptic surface with $\kappa(S)=1$. Assume further that $X$ is not a $\mathbf{P}^{1}$-bundle over a smooth surface $\tilde{S}$ with $S$ as a meromorphic section. Then there is a holomorphic surjection $p: X \rightarrow Y$ onto a smooth curve $Y$ of genus $h^{1,0}(S)$. The smooth fibres of $X$ are rational and for $n>0$ :

$h^{0}\left(\left(K_{X^{\prime}} \otimes\left[S^{\prime}\right]\right)^{n}\right)=h^{0}\left(\left(K_{S^{\prime}}\right)^{n}\right)=n\left(\chi\left(\mathscr{O}_{S^{\prime}}\right)+2 h^{1,0}\left(S^{\prime}\right)-2\right)+1-h^{1,0}\left(S^{\prime}\right)$.

Further $p_{S}: S \rightarrow Y$ is an elliptic fibration, and has no multiple fibres ([B-H], p. 353).

Proof. By (0.6) we can blow down $(X, S)$ to $\left(X^{\prime}, S^{\prime}\right)$.

Claim. $K_{X^{\prime}}+L^{\prime}$ is numerically effective, where $L^{\prime}=\left[S^{\prime}\right]$.

Proof (of claim). Assume that $K_{X^{\prime}}+L^{\prime}$ is not numerically effective, then $K_{X^{\prime}}$ is necessarily not numerically effective. Hence there exists an extremal rational curve $C$ such that $\left(K_{X^{\prime}}+L^{\prime}\right) \cdot C<0$. Since $L^{\prime}$ is ample, $K_{X^{\prime}} \cdot C \leq-2$. But since $C$ is extremal, we also have $K_{X^{\prime}} \cdot C \geq$ -4. Hence $-4 \leq K_{X^{\prime}} \cdot C \leq-2$. Hence by [M, 3.12, 3.39, and 3.39.1], the only possibility is $K_{X^{\prime}} \cdot C=-2$ and $L^{\prime} \cdot C=1$, with $C$ numerically effective (see [M, p. 137]). But $\left(K_{X^{\prime}}+L^{\prime}\right) \cdot C=-2+1=-1$, contrary to the assertion. Hence $K_{X^{\prime}}+L^{\prime}$ must be numerically effective, proving our claim.

In $N E^{-}\left(X^{\prime}\right)$, the curves $C$ such that $\left(K_{X^{\prime}}+L^{\prime}\right) \cdot C=0$, form a face of the subcone $N E_{-}\left(X^{\prime}\right)$ (see [M, (1.5)]). Hence there is a corresponding contraction $p: X^{\prime} \rightarrow Y$ of this face, so that $K_{X^{\prime}}+L^{\prime}=p^{*}(M)$, where $M \in \operatorname{Pic}(Y)$ is ample. But this $p$ contracts elliptic curves in $S^{\prime}$. Hence $\operatorname{dim} p\left(S^{\prime}\right) \leq 1$; since $S^{\prime}$ is ample in $X^{\prime}$, it follows that $p\left(S^{\prime}\right)=Y$. Hence $Y$ is a curve or a point. If $Y$ is a point then $K_{X^{\prime}}+L^{\prime} \cong \mathscr{O}_{X^{\prime}}$, whence $\kappa\left(S^{\prime}\right)=0$, contradicting our assumption on $\kappa\left(S^{\prime}\right)$. Hence $Y$ must be a curve. Hence if $F$ is a general fibre of $p$, then by adjunction $-K_{F}=L_{F}^{\prime}$. Hence the general fibres of $p$ are rational.

The following argument was essentially contained in [So 6]. Since

$$
\left(K_{X^{\prime}}+L^{\prime}\right)=p^{*}(M)
$$

$$
K_{S^{\prime}}=\left(p_{S^{\prime}}\right)^{*}(M)
$$


By the Kodaira vanishing theorem and the Riemann-Roch theorem, for $n>0$ :

$$
\text { (0.7.3) } \begin{aligned}
\chi\left(n\left(K_{X^{\prime}}+L^{\prime}\right)\right)=h^{0}\left(n\left(K_{X^{\prime}}+L^{\prime}\right)\right) \\
=\frac{n}{12}\left(K_{X^{\prime}}+L^{\prime}\right) \cdot\left(2 n\left(K_{X^{\prime}}+L^{\prime}\right)-K_{X^{\prime}}\right)\left(n\left(K_{X^{\prime}}+L^{\prime}\right)-K_{X^{\prime}}\right) \\
\quad+\frac{n}{12}\left(K_{X^{\prime}}+L^{\prime}\right) \cdot c_{2}\left(X^{\prime}\right)+\chi\left(\mathscr{O}_{X^{\prime}}\right) \\
=\frac{n}{12}\left(K_{X^{\prime}}+L^{\prime}\right) \cdot\left((2 n-1)\left(K_{X^{\prime}}+L^{\prime}\right)+L^{\prime}\right) \\
\quad \cdot\left((n-1)\left(K_{X^{\prime}}+L^{\prime}\right)+L^{\prime}\right) \\
\quad+\frac{n}{12}\left(K_{X^{\prime}}+L^{\prime}\right) \cdot c_{2}\left(X^{\prime}\right)+\chi\left(\mathscr{O}_{X^{\prime}}\right) .
\end{aligned}
$$

But we note that $\left(K_{X^{\prime}}+L^{\prime}\right) \cdot\left(K_{X^{\prime}}+L^{\prime}\right) \cdot D=0$, for any effective divisor $D$ on $X^{\prime}$; so

$$
\begin{aligned}
\chi(n & \left.\left(K_{X^{\prime}}+L^{\prime}\right)\right)=h^{0}\left(n\left(K_{X^{\prime}}+L^{\prime}\right)\right) \\
\quad & =\frac{n}{12}\left(K_{X^{\prime}}+L^{\prime}\right) \cdot L^{\prime} \cdot L^{\prime}+\frac{n}{12}\left(-c_{1}\left(X^{\prime}\right)+L^{\prime}\right) \cdot c_{2}\left(X^{\prime}\right)+\chi\left(\mathscr{O}_{X^{\prime}}\right) \\
& =\frac{n}{12} K_{S^{\prime}} \cdot K_{S^{\prime}}+\frac{n}{12} c_{2}\left(S^{\prime}\right)-2 n \chi\left(\mathscr{O}_{X^{\prime}}\right)+\chi\left(\mathscr{O}_{X^{\prime}}\right) \\
& =n\left(\chi\left(\mathscr{O}_{S^{\prime}}\right)-2 \chi\left(\mathscr{O}_{X^{\prime}}\right)\right)+\chi\left(\mathscr{O}_{X^{\prime}}\right) .
\end{aligned}
$$

Since the general fibres of $p$ are rational, we see that $h^{i, 0}\left(X^{\prime}\right)=0$, for $i \geq 2$, and by Lefschetz theorem $h^{1,0}\left(X^{\prime}\right)=h^{1,0}\left(S^{\prime}\right)=g(Y)$.

Hence by (0.7.1) and (0.7.2) we have:

$$
\begin{aligned}
h^{0}\left(p^{*}(M)\right) & =h^{0}\left(\left(K_{X^{\prime}}+L^{\prime}\right)\right) \\
& =h^{0}\left(K_{S^{\prime}}\right)=\chi\left(\mathscr{O}_{S^{\prime}}\right)+g(Y)-1
\end{aligned}
$$

and since $p_{(i)}\left(K_{X^{\prime}}+L^{\prime}\right)=0$; by the Leray spectral sequence,

$$
h^{0}\left(p^{*}(M)\right)=h^{0}(M), \quad \text { and } \quad h^{1}(M)=h^{1}\left(p^{*}(M)\right)=0
$$

by Riemann-Roch on $Y ; h^{0}(M)=\operatorname{deg} M+1-g(Y)$.

Hence by (0.7.4) and (0.7.5)

$$
\chi\left(\mathscr{O}_{S^{\prime}}\right)+2(1-g(Y))=\operatorname{deg} M .
$$

Now by the canonical bundle formula using the notations of ([B-H, p. 353]); for $0 \leq a_{i} \leq m_{i}-1$

$$
\begin{aligned}
\operatorname{det} M= & \operatorname{deg} K_{S^{\prime}}=\chi\left(\mathscr{O}_{S^{\prime}}\right)+2(1-g(Y)) \\
& + \text { length } T+\sum_{i}\left(a_{i} / m_{i}\right) .
\end{aligned}
$$

Hence by (0.7.6) and (0.7.7) it follows that length $T=0$, and $a_{i}=0$, for all $i$. Hence all the fibres of $p_{S^{\prime}}$ are non-multiple and reduced, and we are done. 
(0.8) THEOREM. Let $S$ be a smooth ample divisor on a connected projective threefold $X$, then $\log (X, S)<0$ if and only if one of the following is true.

(a) $X$ is a $\mathbf{P}^{1}$-bundle over a smooth surface $\tilde{S}$ and $S$ is a meromorphic section,

(b) $S$ is of negative Kodaira dimension.

(0.9) THEOREM. Let $L$ be an ample line bundle on a smooth connected surface $S$. Then $\Gamma\left(\left(K_{S} \otimes L\right)^{n}\right)$ spans $\left(K_{S} \otimes L\right)^{n}$, for some $n>0$, unless $(S, L)$ is of the form:

(a) $\left(\mathbf{P}^{2}, \mathscr{O}_{\mathbf{P}^{2}}(e)\right)$ with $e=1$ or 2 ,

(b) $S$ is a $\mathbf{P}^{1}$-bundle over a curve and $L_{F} \cong \mathscr{O}_{\mathbf{P}^{1}}(1)$, for any fibre $F$ of the bundle.

(0.10) REMARK. $\log (X, S)=1$ if and only if $(X, S)$ satisfies Theorem (0.7), and from the proof of this theorem, we have:

(1) $K_{X^{\prime}} \otimes\left[S^{\prime}\right]=p^{*}(M)$, where $M$ is a line bundle over $Y$.

(2) $K_{X^{\prime}} \otimes\left[S^{\prime}\right]$ is arithmetically effective.

(0.11) Remark. Proving a theorem for $\left(X^{\prime}, L^{\prime}\right)$ implies the conclusions of the theorem for $(X, L)$.

\section{Some general results.}

(1.1) Definition. Let $p: X \rightarrow Y$ be a surjective morphism, where $X$ is a smooth projective manifold and $Y$ is a smooth curve. Let $\mathscr{E}$ be a holomorphic line bundle on $X$. Suppose also that for all $y$ in $Y, \mathscr{E}$ restricted to $p^{-1}\left(\Delta_{y}\right)$ is spanned (ample, very ample) where $\Delta_{y}$ is a neighborhood of $y$. Then $\mathscr{E}$ is said to be locally spanned (ample, very ample), with respect to $p$.

(1.2) Proposition. Let $p: X \rightarrow Y$ be a holomorphic map, where $X$ is a smooth projective threefold and $Y$ is a smooth curve. Let $\mathscr{E}$ be a holomorphic line bundle on $X$, such that $p_{*}(\mathscr{E})$ is locally free of rank $(r+1)$ on $Y$. Suppose also that $\mathscr{E}$ is locally spanned with respect to $p$. Then there exists a holomorphic map $\Phi: X \rightarrow \mathbf{P}\left(p_{*}(\mathscr{E})\right)$, such that the following diagram of morphisms commutes:

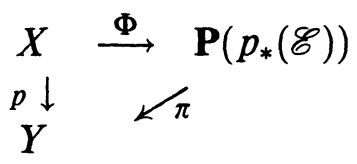

Proof. The proof of the above proposition is a formal exercise. 
(1.3) COROLlARY. With the same hypothesis as in (1.2), if moreover, $\mathscr{E}$ is locally very ample with respect to $p$, then $\Phi: X \rightarrow \mathbf{P}\left(p_{*}(\mathscr{E})\right)$ is a relative embedding.

Proof. We choose an open cover $\left\{U_{i}\right\}$ of $Y$, where $U_{i}$ is chosen small enough to have $p_{*}(\mathscr{E})$ free of rank $(r+1)$ over $U_{i}$. Let $U_{i j}$ denote the intersection of $U_{i}$ and $U_{j}$. Since $\Phi_{i}$ is an embedding for all $i$, then we have that $\Phi_{i j}: p^{-1}\left(U_{i j}\right) \rightarrow U_{i j} \times \mathbf{P}^{r}$ is also an embedding. Hence $\Phi^{*}$ induces an embedding of $X$ in $\mathbf{P}\left(p_{*}(\mathscr{E})\right)$.

(1.4) Lemma. Let $p: X^{\prime} \rightarrow Y$ be a proper morphism, where $X^{\prime}$ is a smooth projective threefold and $Y$ a smooth curve. Let $L^{\prime}$ be an ample line bundle with respect to $p$, where $\left(X^{\prime}, L^{\prime}\right)$ is as in (0.6.1). Then $p_{*}\left(-K_{X^{\prime}}\right)$ is locally free everywhere of constant rank.

Proof. $p_{*}\left(-K_{X^{\prime}}\right)$ is torsion free and $Y$ is smooth. Hence $p_{*}\left(-K_{X^{\prime}}\right)$ is everywhere free of the same rank as that of this sheaf in the neighborhood of a smooth fibre.

(1.4.1) LemMA. Under the conditions of Lemma (1.4), $p_{(i)}\left(\mathscr{O}_{X^{\prime}}\right)=0$ and $p_{(i)}\left(\mathscr{O}_{X^{\prime}}(-F)\right)=0$, where $F$ is any fibre of $p$ and $i>0$.

Proof. By (0.11), $K_{X^{\prime}}+L^{\prime}=p^{*}(M)$, so using $-M$ in [So 1, p. 60, Lemma II-A], $p_{(i)}\left(\mathscr{O}_{X^{\prime}}\right)=0$.

Now noting that $\mathscr{O}_{X^{\prime}}(-F)=p^{*}(-[P])$, where $p^{-1}(-P)=F$, we can rewrite, again using $(0.11), \mathscr{O}_{X^{\prime}}(-F)=p^{*}(-M-[P])+K_{X^{\prime}}+L^{\prime}$ and again using the same lemma we see that $p_{(i)}\left(\mathscr{O}_{X^{\prime}}(-F)\right)=0$.

(1.5) REMARK. By Theorem (0.7) $\mathscr{O}_{F} \cong\left(p^{*}(-M)\right)_{F} \otimes\left(K_{F} \otimes L_{F}\right)$, where $M$ is a holomorphic line bundle on $Y$, and $F$ is a general fibre of $p$. Hence for a general fibre of $p,\left(K_{F}\right)^{-1}$ is ample in $F$. Hence $F$ is a del Pezzo surface. We denote the degree of the del Pezzo surface by $r$, where $r=K_{F} \cdot K_{F}$.

We need the following lemmas.

(1.6) Lemma. Let $(X, L)$ be as in (0.6), with $L$ very ample. Given two distinct points $\{x, y\}$ in $X$, there is a smooth $A \in|L|$ with $\{x, y\}$ in $A$. Given a point $x$ and a tangent vector $v$ at $x$, there is a smooth $A \in|L|$, with $x$ in $A$, and $v \in T_{A}$, where $T_{A}$ is the tangent bundle of $A$.

Proof. If the intersection of all $A \in|L|$ that contain $\{x, y\}$ is a finite set $G$, then by Bertini's theorem and the fact that a generic $A \in|L|$ 
containing $g \in G$, is smooth at $g$, we can find such a smooth $A$. On the other hand if the intersection of all $A \in|L|$ that contain $\{x, y\}$ is not a finite set then since $\Gamma(L)$ embeds $X$ in $\mathbf{P}$, it follows that the intersection must contain a line $h$. Let $\mathscr{J}_{h}$ denote the ideal sheaf of $h$ in $X$. Since the zero set of the general section of $L \otimes \mathscr{J}_{h}$ gives rise to a non-zero section of $\left(\mathscr{J}_{h} / \mathscr{J}_{h}^{2}\right) \otimes L$, it follows that the zero set $A$ of the general section of $L \otimes \mathscr{J}_{h}$ is smooth along $h$. Hence again by Bertini's theorem $A$ is smooth. A similar argument holds in the case $x \in X$, and $v$ a tangent vector at $X$.

(1.6.1) LEMMA. If $L$ is very ample then $L^{\prime}$ is locally very ample with respect to $p$.

Proof. By (a) in Theorem (0.6) we have $\pi^{*}\left(K_{X^{\prime}} \otimes L^{\prime 2}\right) \cong K_{X} \otimes L^{2}$ and $L^{\prime}$ is ample. See [So 2] for details. Also from the exact sequence:

$$
0 \rightarrow K_{X} \otimes L \rightarrow K_{X} \otimes L^{2} \rightarrow K_{S} \otimes L_{S} \rightarrow 0
$$

and using the fact that $\pi_{(1)}\left(K_{X} \otimes L\right)=0$, we have

$$
K_{X^{\prime}} \otimes L^{\prime 2} \rightarrow \pi_{*}\left(K_{S} \otimes L_{S}\right) \rightarrow 0 .
$$

Also since $S$ is an elliptic surface of non-negative Kodaira dimension, it is non-ruled in particular. Hence by [So 3] $K_{S} \otimes L_{S}$ is spanned. Also note that:

(*) $\quad K_{X^{\prime}} \otimes L^{\prime}=p^{*}(\mathscr{M})$, with $\operatorname{deg}(\mathscr{M})>0$, since $\log (X, S)=1$.

Since $L$ is very ample, by [So 4]

$K_{X^{\prime}} \otimes L^{\prime 2}$ is spanned by global sections.

Hence by $(*)$ and $(* *) L^{\prime} \otimes p^{*}(\mathscr{M})$ is spanned. Hence $L^{\prime}$ is locally spanned. Now by $(1.6)$ it suffices to show that $L_{\mid S^{\prime}}^{\prime}$ is locally very ample.

Claim. $L_{S} \cdot L_{S} \geq 6$.

Proof (of claim). Since $L_{S}$ is very ample and $K_{S} \otimes L_{S}$ is spanned,

$$
g\left(L_{S}\right) \neq h^{1,0}(S) \quad(\text { see } 1.5 \text { in [So 3]). }
$$

If $L_{S} \cdot L_{S} \leq 5$, then by Castelnuovo's inequality, [Ha 2] we have $g \leq 2$. But by [So 3, Lemma 2.2.2 for $g=2$, and for $g=1, h^{1,0}(S)=$ $0]$, it follows that the Kodaira dimension of $S$ is negative, but we have $\kappa(S) \geq 0$.

Hence $L_{S} \cdot L_{S} \geq 6$, proving the claim. 
From the exact sequence:

$$
0 \rightarrow K_{X^{\prime}} \otimes L^{\prime} \rightarrow K_{X^{\prime}} \otimes L^{\prime 2} \rightarrow K_{S^{\prime}} \otimes L_{S^{\prime}}^{\prime} \rightarrow 0 .
$$

On tensoring the above sequence with $\left(K_{X^{\prime}} \otimes L^{\prime}\right)^{n-1}$ we see that by the Kodaira vanishing theorem, $H^{i}\left(X^{\prime},\left(K_{X^{\prime}} \otimes L^{\prime}\right)^{n}\right)=0$ for $i>0$. Hence

$$
\Gamma\left(\left(K_{X^{\prime}} \otimes L^{\prime}\right)^{n} \otimes L^{\prime}\right) \rightarrow \Gamma\left(\left(K_{S^{\prime}}\right)^{n} \otimes L_{S^{\prime}}^{\prime}\right) \rightarrow 0 .
$$

Hence the sections of $\left(K_{S^{\prime}}\right)^{n} \otimes L^{\prime}$ lift to sections of $\left(K_{X^{\prime}} \otimes L^{\prime}\right)^{n} \otimes L^{\prime}$. Since $\kappa\left(S^{\prime}\right)$ is non-negative $\left(K_{S^{\prime}}\right)^{n} \neq 0$, for some $n>0$, so $L \otimes \pi^{*}\left(\left(K_{S^{\prime}}\right)^{n}\right)$ is very ample on $S$, where $\pi$ is as in (0.6). So by [VdV Theorem III] $K_{S} \otimes L \otimes \pi^{*}\left(\left(K_{S^{\prime}}\right)^{n}\right)$ is very ample on $S$. Hence $\pi_{*}\left(K_{S} \otimes L \otimes \pi^{*}\left(\left(K_{S^{\prime}}\right)^{n}\right)\right)$ separates points on $S^{\prime}$. But $K_{S^{\prime}}$ is locally trivial since $S^{\prime}$ is minimally elliptic (see [B-H], p. 353). Hence $L_{S^{\prime}}^{\prime}$ separates points locally. Since $S^{\prime}$ is obtained by blowing down finitely many exceptional curves on $S, L_{S^{\prime}}^{\prime}$ separates tangent vectors. Hence $L_{S^{\prime}}^{\prime}$ is locally very ample.

(1.6.2) Lemma. Let $L$ be ample and spanned over $X$, then $L^{\prime}$ is locally ample and spanned.

Proof. By (0.6) $L^{\prime}$ is ample, and by (1.6.1) we also know that $L_{S} \cdot L_{S} \geq 6$. We first show that $K_{S^{\prime}} \otimes L_{S^{\prime}}$ is spanned by global sections. We have the following exact sequences:

$$
\begin{aligned}
& 0 \rightarrow K_{X} \otimes L \rightarrow K_{X} \otimes L^{2} \rightarrow K_{S} \otimes L_{S} \rightarrow 0 \\
& 0 \rightarrow K_{X} \otimes L \quad \rightarrow \quad K_{X} \otimes L^{2} \quad \rightarrow \quad K_{S} \otimes L_{S} \rightarrow 0 \\
& \pi^{* \downarrow} \quad \pi^{*} \downarrow \\
& 0 \rightarrow K_{X^{\prime}} \otimes L^{\prime} \rightarrow K_{X^{\prime}} \otimes L^{\prime 2} \rightarrow K_{S^{\prime}} \otimes L_{S^{\prime}}^{\prime} \rightarrow 0 .
\end{aligned}
$$

Since $\pi$ blows down exceptional curves of $S, \pi_{(i)}\left(K_{S} \otimes L_{S}\right)=0$ (see (0.5.4)), for $i>0$. Hence by the Leray spectral sequence

$$
\Gamma\left(S, K_{S} \otimes L_{S}\right) \cong \Gamma\left(S^{\prime}, K_{S^{\prime}} \otimes L_{S^{\prime}}\right) .
$$

Since $L_{S} \cdot L_{S} \geq 6$, and $L_{S}$ is ample and spanned and $S$ has nonnegative Kodaira dimension, it follows by [So 5] that $K_{S} \otimes L_{S}$ is spanned. Hence $K_{S^{\prime}} \otimes L_{S^{\prime}}^{\prime}$ is spanned. Now considering the long exact sequence associated with

$$
0 \rightarrow K_{X^{\prime}} \otimes L^{\prime} \rightarrow K_{X^{\prime}} \otimes L^{\prime 2} \rightarrow K_{S^{\prime}} \otimes L_{S^{\prime}}^{\prime} \rightarrow 0
$$

and noting that $H^{1}\left(X^{\prime}, K_{X^{\prime}} \otimes L^{\prime}\right)=0$ by the Kodaira vanishing theorem, we see that

$$
\Gamma\left(K_{X^{\prime}} \otimes L^{\prime 2}\right) \rightarrow \Gamma\left(K_{S^{\prime}} \otimes L_{S^{\prime}}^{\prime}\right) \rightarrow 0 .
$$


Hence $K_{X^{\prime}} \otimes L^{\prime 2}$ is spanned by global sections. But $K_{X^{\prime}} \otimes L^{\prime}$ is locally trivial. Hence $L^{\prime}$ is locally spanned.

\section{(1.7) Possible Singular Fibres of $p_{S^{\prime}}$}

Following (0.7) we have:

$$
\mathscr{O}_{F} \cong p^{*}(\mathscr{M})_{F} \cong\left(K_{F} \otimes L_{F}^{\prime}\right)^{N}
$$

where $\mathscr{M}$ is a holomorphic line bundle over $Y . L^{\prime}$ is the line bundle given by $S^{\prime}$ in $X^{\prime}, F$ is a general fibre of $p$, and $N$ is some integer.

By (1.7.1) $\left(K_{F}\right)^{-1}$ is ample in $F$, hence $F$ is a del Pezzo surface and in particular $F$ is rational. We first calculate $\Sigma \cdot S^{\prime}$ on $X^{\prime}$, where $\Sigma$ is singular fibre of $p_{\mid S^{\prime}}$. Since $\Sigma \sim E$ on $S^{\prime}$ where $E$ is a smooth elliptic curve on $S^{\prime}$; we have $\Sigma \cdot S^{\prime}=E \cdot S^{\prime}$ in $X^{\prime}$ and also $E \cdot S^{\prime}=(E \cdot E)_{F}$, and since $E=S^{\prime} \cdot F, E$ is ample in $F$. Also by (1.7.1) $E \cdot E=$ $\left(K_{F}\right)^{-1} \cdot\left(K_{F}\right)^{-1}$. Hence $\Sigma \cdot S^{\prime}=K_{F} \cdot K_{F}$. But for del Pezzo surfaces we know that $K_{F} \cdot K_{F} \leq 9$. Hence we have $1 \leq \Sigma \cdot S^{\prime} \leq 9$. Hence the reduced singular fibres of $p_{S^{\prime}}$ can have at most 9 components. So the only possible ones by [Ko 1] are of type $\mathrm{I}_{b}, 1 \leq b \leq 9$, II, III, IV and $\mathrm{I}_{b}^{*}, b=0,1$. We also note that $\Sigma \cdot S^{\prime} \geq 3$ if $S^{\prime}$ is very ample.

(1.8) THEOREM. Let $\left(X^{\prime}, L^{\prime}\right)$ be as in (0.6.1) and assume that $L$ is ample and spanned over $X$, then there exists a morphism $\Phi: X^{\prime} \rightarrow$ $\mathbf{P}\left(p_{*}\left(-K_{X^{\prime}}\right)\right)$ such that $\mathbf{P}\left(p_{*}\left(-K_{X^{\prime}}\right)\right)$ is a $\mathbf{P}^{r}$-bundle over $Y$, where $r$ is the degree of the del Pezzo surface that is the general fibre of $p: X^{\prime} \rightarrow Y$. Moreover we have the following commutative diagram of morphisms:

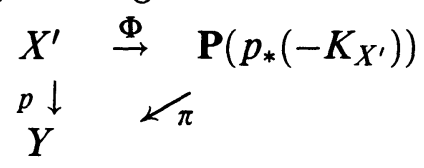

Proof. By Remark (1.5) we know that the general fibre of $p: X^{\prime} \rightarrow Y$ is a del Pezzo surface of degree $r$, where $r=K_{F} \cdot K_{F}$. Now by looking in the neighborhood of the general fibre we see that $p_{*}\left(-K_{X^{\prime}}\right)$ is locally free of rank $(r+1)$. Hence by Lemma (1.4) it is everywhere free of rank $(r+1)$. The rest of the theorem is an immediate consequence of Propositions (1.2) and (1.6.2).

2. The different cases of $r=K_{F} \cdot K_{F}$.

(2.0) In what follows $\left(X^{\prime}, S^{\prime}\right)$ and $\left(X^{\prime}, L^{\prime}\right)$ are as in (0.6.1). Moreover we assume that $S$ is ample in $X$, and $p: X^{\prime} \rightarrow Y$ is as in Lemma (1.4).

(A) The case $d=2$.

Here we need the following classical result. 
(2.1.0) Proposition. A smooth del Pezzo surface of degree 2 is a double cover of $\mathbf{P}^{2}$, branched along a smooth quartic curve in $\mathbf{P}^{2}$.

Remark. Note that the singularities of the del Pezzo surface lie exactly at the place where branching occurs in (2.1.0).

(2.1.1) Theorem. Let $p, X^{\prime}, S^{\prime}$ and $Y$ be as in (2.0). Assume further that $L$ is spanned. Then there exists a commutative diagram of morphisms:

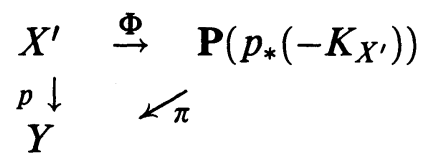

such that $\boldsymbol{\Phi}$ is a $2: 1$ cover of $\mathbf{P}\left(p_{*}\left(-K_{X^{\prime}}\right)\right)$ branched along a surface that intersects every fibre of $\pi$ along a quartic curve (not necessarily smooth).

Proof. We first note that $p_{*}\left(-K_{X^{\prime}}\right)$ is locally free of rank 3. Hence by Propositions (1.2) and (1.6.2), there exists a morphism $\Phi: X \rightarrow$ $\mathbf{P}\left(p_{*}\left(-K_{X^{\prime}}\right)\right)$.

Now by (2.1.0), it follows that $\Phi$ is $2: 1$ everywhere along the smooth fibres of $p$, and along the singular fibres, by the remark and noting that there are only finitely many singular fibres, it follows that $\Phi$ is $2: 1$ almost everywhere except along some isolated points. But this being a set of codimension $2, \Phi$ extends over to be 2 : 1 everywhere.

(2.1.2) THEOREM. If $X^{\prime}$ is a smooth projective threefold, $Y$ a smooth curve, and $\mathbf{P}(\mathscr{V})$ a $\mathbf{P}^{2}$-bundle over $Y$, such that there is a $2: 1$ morphism $\Phi: X^{\prime} \rightarrow \mathbf{P}(\mathscr{V})$, branched along a surface, whose general fibre via the restriction map of $\Phi$, is a quartic curve, then the general fibre of $p$ (as in (2.0)) must be a del Pezzo surface of degree 2.

Proof. Since $\Phi$ is $2: 1$ over $\mathbf{P}(\mathscr{V})$, it follows that the pullback of an ample divisor is again ample. Let $\phi=\left.\Phi\right|_{F}$ where $F$ denotes a general fibre of $p$, then

$$
K_{F}=\phi^{*}\left(K_{\mathbf{P}^{2}}\right)+B=\phi^{*}(-3 H)+B
$$

where $H$ is the hyperplane in $\mathbf{P}^{2}$, and $B$ is the branch locus. Also $2 B=\phi^{*}(4 H)$. Hence $B=\phi^{*}(2 H)$, so that $-K_{F}=\phi^{*}(H)$. Thus $-K_{F}$ is ample in $F$, and since $\Phi$ is $2: 1, \operatorname{deg}\left(-K_{F}\right)=2$. So $F$ is a del Pezzo surface of degree 2 .

REMARK. The case $r=2$ cannot occur if $S$ if very ample in $X$. 
(B) The case $r=3$.

(2.2.1) Theorem. Let $p, X^{\prime}, S^{\prime}$ and $Y$ be as in (2.0). Assume moreover that $S$ is very ample in $X$. Then there exists a morphism $\Phi: X^{\prime} \rightarrow$ $\mathbf{P}\left(p_{*}\left(-K_{X^{\prime}}\right)\right)$ such that $\Phi$ is an embedding in the sense of (1.3). Moreover we have the following commutative diagram of morphisms:

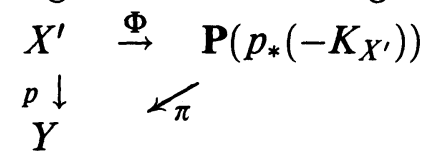

where $\mathbf{P}\left(p_{*}\left(-K_{X^{\prime}}\right)\right)$ is a $\mathbf{P}^{3}$-bundle over $Y$.

Proof. This is an immediate consequence of (1.6.1) and (1.8).

Let $\mathscr{V}=p_{*}\left(-K_{X^{\prime}}\right)$. Since in the above $\Phi$ is an embedding, $X^{\prime}$ can be thought of as a divisor in $\mathbf{P}(\mathscr{V})$. Hence noting that $\pi_{*}\left(\xi^{3}\right) \cong \mathscr{S}^{3}(\mathscr{V})$, where $\xi$ is the tautological bundle, and since $\left.X^{\prime}\right|_{\pi^{-1}(y)}$ is a smooth cubic for a generic $y \in Y$, we have

$$
\pi_{*}\left(\left.\left[X^{\prime}\right]\right|_{\pi^{-1}(y)}\right) \cong \mathscr{S}^{3}(\mathscr{V})_{y} .
$$

Hence

$$
\mathbf{P}\left(\pi_{*}\left(\left[X^{\prime}\right]\right)\right)=\mathbf{P}\left(\mathscr{S}^{3}(\mathscr{V})\right)
$$

Therefore

$$
\pi_{*}\left(\left[X^{\prime}\right]\right) \cong \mathscr{S}^{3}(\mathscr{V}) \otimes \mathscr{M}^{\prime}
$$

for some line bundle $\mathscr{M}^{\prime}$ on $Y$. So it follows by the projection formula that

$$
\pi_{*}\left(\left[X^{\prime}\right]\right) \cong \pi_{*}\left(\xi^{3} \otimes \pi^{*}\left(\mathscr{M}^{\prime}\right)\right)
$$

or

$$
\left[X^{\prime}\right]=\left[\xi^{3} \otimes \pi^{*}\left(\mathscr{M}^{\prime}\right)\right]
$$

This brings us to the converse of (2.2.1).

(2.2.2) Theorem. If $\pi: \mathbf{P}(\mathscr{V}) \rightarrow Y$ is a morphism, where $Y$ is a smooth curve and $\mathbf{P}(\mathscr{V})$ is a $\mathbf{P}^{3}$-bundle over $Y$, and $X^{\prime}$ is embedded in $\mathbf{P}(\mathscr{V})$ such that $X^{\prime} \in\left|\xi^{3} \otimes \pi^{*}\left(\mathscr{M}^{\prime}\right)\right|$, where $\xi$ and $\mathscr{M}^{\prime}$ are as above, then $\pi_{X^{\prime}}: X^{\prime} \rightarrow Y$ is a del Pezzo fibering with the general fibres as cubic surfaces in $\mathbf{P}^{3}$.

Proof.

$$
\begin{aligned}
K_{X^{\prime}} & =\left(K_{\mathbf{P}(\mathscr{V})} \otimes\left[X^{\prime}\right]\right)_{\mid X^{\prime}} \quad \text { by adjunction } \\
& =\left(\xi^{-4} \otimes \pi^{*}\left(K_{Y} \otimes \operatorname{det}(\mathscr{V})\right) \otimes \xi^{3} \otimes \pi^{*}\left(\mathscr{M}^{\prime}\right)\right)_{\mid X^{\prime}} \\
& =\xi_{\mid X^{\prime}}^{-1} \otimes \pi^{*}\left(K_{Y} \otimes \operatorname{det}(\mathscr{V}) \otimes \mathscr{M}^{\prime}\right)_{\mid X^{\prime}}
\end{aligned}
$$


where $\operatorname{det}(\mathscr{V})$ is the determinant bundle of $\mathscr{V}$. Hence for any general fibre $F$ of $\pi_{X^{\prime}}$, we have:

$$
K_{F}=\left.K_{X^{\prime}}\right|_{F}=\left.\left(\xi^{-1}\right)_{X^{\prime}}\right|_{F} \otimes \pi^{*}\left(K_{Y} \otimes \operatorname{det}(\mathscr{V}) \otimes \mathscr{M}^{\prime}\right)_{\mid X}
$$

thus $\left(K_{F}\right)^{-1}$ is very ample in $F$. Since $X^{\prime \prime}\left|\xi^{3} \otimes \pi^{*}\left(\mathscr{M}^{\prime}\right)\right|$, it must be a del Pezzo surface of degree 3 in $\mathbf{P}^{3}$.

(2.2.3) REMARK. From the proof of (2.2.2), since $Y$ is smooth it follows that $K_{Y} \otimes \operatorname{det}(\mathscr{V}) \otimes \mathscr{M}=\mathscr{O}_{Y}$.

(2.2.4) CoRollary. In (2.2.2), if $\left[\xi^{3} \otimes \pi^{*}\left(K_{Y} \otimes \operatorname{det}(\mathscr{V})\right)\right]$ is nonempty and ample then $\Phi\left(X^{\prime}\right)$ is ample and $H^{2}\left(X^{\prime}, \mathbf{Z}\right) \cong \mathbf{Z} \oplus \mathbf{Z}$.

Proof. Since ample line bundles restrict, $\Phi\left(X^{\prime}\right)$ is ample, and since $\operatorname{dim} \mathbf{P}(\mathscr{V})$ is 4 , by Lefschetz theorem $H^{2}\left(X^{\prime}, \mathbf{Z}\right) \cong H^{2}(\mathbf{P}(\mathscr{V}), \mathbf{Z}) \cong$ $\mathbf{Z} \oplus \mathbf{Z}$.

(C) The case $r=4$.

Before proceeding, we need the following classical result.

(2.3.0) Proposition. A smooth del Pezzo surface of degree 4 can be embedded as a complete intersection of two smooth quadric hypersurfaces in $\mathbf{P}^{4}$.

(2.3.1) Theorem. Assume $S$ is very ample in $X$, and let $p, X^{\prime}, S^{\prime}$ and $Y$ be as in (2.0). Then there exists a morphism $\Phi: X^{\prime} \rightarrow \mathbf{P}\left(p_{*}\left(-K_{X^{\prime}}\right)\right)$ such that $\Phi$ is an embedding in the sense of (1.3). Moreover we have the following commutative diagram of morphisms:

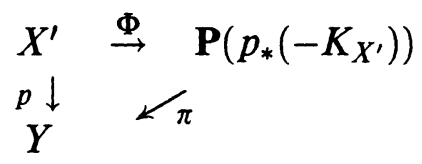

where $\mathbf{P}\left(p_{*}\left(-K_{X^{\prime}}\right)\right)$ is a $\mathbf{P}^{4}$-bundle over $Y$, and $\boldsymbol{\Phi}\left(X^{\prime}\right)$ is a complete intersection of two divisors (which are unique upto a pullback from the base), in a small neighborhood of every fibre of $p$.

Proof. By (1.6.1) and (1.8) we have the embedding and the commutativity of the diagram of morphisms. If $F$ is a smooth fibre of $p$, then by (2.3.0) we can always find smooth quadrics $\mathscr{Q}_{0}$ and $\mathscr{Q}_{1}$ in $\mathbf{P}^{4}$, containing $F$. Hence the fibre of $\pi$ contains a pencil of quadrics intersecting in $F$. If we denote this linear system by $\left|\mathscr{Q}^{\lambda}\right|=\left|\lambda_{0} \mathscr{Q}_{0}+\lambda_{1} \mathscr{Q}_{1}\right|$, 
then by Bertini's theorem we can always find a non-singular $\mathscr{Q}^{\lambda}$ containing $F$.

Claim. This holds true even for singular fibres.

Proof (of claim). Let $\xi$ be the tautological line bundle of $\mathbf{P}(\mathscr{V}), \mathscr{V}=$ $p_{*}\left(-K_{X^{\prime}}\right)$, and $\mathscr{J}_{X^{\prime}}$ the ideal sheaf of $X^{\prime}$ in $\mathbf{P}(\mathscr{V})$. Then we have the following short exact sequence:

$$
\left.0 \rightarrow \xi^{2} \otimes \mathscr{J}_{X^{\prime}} \rightarrow \xi^{2} \rightarrow \xi^{2}\right|_{X^{\prime}} \rightarrow 0
$$

which gives rise to the following long exact sequence:

$$
\cdots \rightarrow \pi_{*}\left(\xi^{2}\right) \rightarrow \pi_{*}\left(\left.\xi^{2}\right|_{X^{\prime}}\right) \rightarrow \pi_{(1)}\left(\xi^{2} \otimes \mathscr{J}_{X^{\prime}}\right) \rightarrow 0
$$

noting that $\pi_{*}\left(\xi^{2}\right) \cong \mathscr{S}^{2}(\mathscr{V})$, and so has rank 15 , and since a smooth fibre of $p$ is a local complete intersection of two quadrics in $\mathbf{P}^{4}$, by (2.3.0), it follows that $\pi_{*}\left(\left.\xi^{2}\right|_{X^{\prime}}\right)$ is locally free of rank 13 in the neighborhood of this smooth fibre. But by (1.4) it is everywhere of the same rank 13. Since the problem is local we let $y_{0} \in Y$ be a point over which the fibre of $p$ is singular, say $S_{0}$. Since $\Phi$ is an embedding, we think of $S_{0}$ in $\mathbf{P}(\mathscr{V})$. Let $\Delta=\Delta_{y_{0}}$ be a small neighborhood of $y_{0}$, chosen small enough so that $\left.\pi_{*}\left(\xi^{2}\right)\right|_{\Delta}$ is free of rank 15 , and $\left.\pi_{*}\left(\left.\xi^{2}\right|_{X^{\prime}}\right)\right|_{\Delta}$ is free of rank 13 . So locally at $\Delta$, we have the following exact sequence:

$$
0 \rightarrow \mathscr{K} \rightarrow \pi_{*}\left(\xi^{2}\right) \stackrel{\beta}{\rightarrow} \pi_{*}\left(\xi_{\mid X^{\prime}}^{2}\right) \rightarrow \pi_{(1)}\left(\xi^{2} \otimes \mathscr{J}_{X^{\prime}}\right) \rightarrow \cdots
$$

where $\mathscr{K}$ is the kernel of $\beta$. Now $\mathscr{K}$ is at least of rank 2 , and so $S_{0}$ is at least contained in an intersection of two quadric hypersurfaces. We need to show that $\mathscr{K}$ is generated by exactly two elements, from which it will follow that locally $\beta$ is surjective. Now let $S_{0}$ lie in say $\mathscr{Q} \cap \mathscr{Q}^{\prime}$ in $\pi^{-1}\left(y_{0}\right) \cong \mathbf{P}^{4}$. We note that $\operatorname{deg}\left(S_{0}\right)=4$. So we have the following possibilities:

(i) Both $\mathscr{Q}$ and $\mathscr{Q}^{\prime}$ are irreducible

(ii) $\mathscr{Q}$ is irreducible but $\mathscr{Q}^{\prime}$ is not

(iii) $\mathscr{Q}$ and $\mathscr{Q}^{\prime}$ are both irreducible without a common component

(iv) $\mathscr{Q}$ and $\mathscr{Q}^{\prime}$ are both reducible having one linear factor in common (v) $\mathscr{Q}=\mathscr{Q}^{\prime}=L_{1} \cup L_{2}$ where $L_{i}$ is a linear factor

We first note that since the degree of the general fibre is 4 , the only possible fibres of $p_{S^{\prime}}$ are of type $\mathrm{I}_{b}$, where $b \leq 4$, and type II, III and IV. We also note that none of these have components of multiplicity $>1$. This is because $L^{\prime} \cap S_{0}$ is reduced (see 0.7 ). Hence the fibre of $p$ cannot have components of multiplicity $>1$, because otherwise $L^{\prime}$ 
will intersect $S_{0}$ in a non-reduced fibre. We now show that none of (i), (ii), (iii), (iv) and (v) are possible.

In (i), (ii) and (iii), $\operatorname{deg}\left(\mathscr{Q} \cap \mathscr{Q}^{\prime}\right)=4=\operatorname{deg}\left(S_{0}\right)$, and $S_{0}$ is reduced. Hence $S_{0}=\mathscr{Q} \cap \mathscr{Q}^{\prime}$.

In (iv) let $\mathscr{Q}=L \cap L_{1}$ and $\mathscr{Q}^{\prime}=L \cap L_{2}$, then $\mathscr{Q} \cap \mathscr{Q}^{\prime}=L \cup\left(L_{1} \cap L_{2}\right)$. Now $L \cong \mathbf{P}^{3}$ and $L_{1} \cap L_{2} \cong \mathbf{P}^{2}$. And $S_{0}$ is in $\mathbf{P}^{3} \cup \mathbf{P}^{2}$, and we have the following possibilities:

(a) $L_{1} \cap L_{2}$ is not a component of $S_{0}$, in which case, $S_{0}$ is in $\mathbf{P}^{3}$.

(b) $L_{1} \cap L_{2}$ is a component of $S_{0}$, whereby $S_{0}=S_{1} \cup S_{2}, S_{1}$ is in $\mathbf{P}^{3}$, and $S_{2}$ is in $\mathbf{P}^{2}, \operatorname{deg}\left(S_{1}\right)=3, \operatorname{deg}\left(S_{2}\right)=1$.

In (a), the general hyperplane in $\pi^{-1}\left(y_{0}\right) \cap X^{\prime}$, given by the line bundle $L^{\prime}$, will intersect $S_{0}$ in a plane quartic curve, say $C$. But then $\chi\left(\mathscr{O}_{C}\right) \neq 0$, and since $\chi\left(\mathscr{O}_{C}\right)=0$, for a general fibre of $p_{S^{\prime}}$, we have a contradiction.

In (b), since $\operatorname{deg}\left(S_{1}\right)=3$, and $\operatorname{deg}\left(S_{2}\right)=1$, the general hyperplane section given by $L^{\prime}$ will possibly intersect $S_{0}$ as follows:

(i) $S_{1}$ in an irreducible cubic, and $S_{2}$ in a line. But in [Ko 1], no such configuration occurs.

(ii) $S_{1}$ in a reducible cubic (conic + line, or, three lines) and $S_{2}$ in a line. Upon examining all possible configurations, we see again by [Ko 1], that none of them occur.

Case (v) cannot occur for then rank of $\mathscr{K}$ will be less than 2 . Hence none of the above cases occur, thus making $\pi_{*}\left(\left.\xi^{2}\right|_{X^{\prime}}\right)$ locally free of rank 13. Hence at $y_{0}, \mathscr{K}$ has rank 2 . This holds for all singular fibres which are finitely many. Hence $\Phi\left(X^{\prime}\right)$ is a local complete intersection in the neighborhood of every fibre.

(2.3.2) COROLlaRY. If $Y=\mathbf{P}^{1}$, then $\Phi\left(X^{\prime}\right)$ is always a complete intersection.

Proof. We know that if we are over $\mathbf{P}^{1}$, then $\pi_{*}(\xi)$ splits into a direct sum of line bundles. So considering the generators of each as being inside $\pi_{*}\left(\xi^{2}\right), \beta$ in (2.3.1) will be surjective, thereby showing that $\Phi\left(X^{\prime}\right)$ is always a complete intersection.

(2.3.3) Proposition. Conversely if $\pi: \mathbf{P}(\mathscr{V}) \rightarrow Y$ is a morphism, where $\mathbf{P}(\mathscr{V})$ is a $\mathbf{P}^{4}$-bundle over $Y$, and $X^{\prime}=\mathscr{Q}_{0} \cap \mathscr{Q}_{1}$ as a complete intersection in $\mathbf{P}(\mathscr{V})$, where $\mathscr{Q}_{i} \in\left|\xi^{2} \otimes \pi^{*}\left(M_{i}\right)\right|, M_{i}$ are line bundles over $Y$, and $\xi=\mathscr{O}_{\mathbf{P}(\mathscr{V})}(1)$ is the tautological bundle. Then $\pi_{X^{\prime}}: X^{\prime} \rightarrow Y$ is a del Pezzo fibering whose general fibres are of degree 4. 
Proof. By adjunction $K_{X^{\prime}}=\left.\left.K_{\mathscr{C}_{0}}\right|_{X^{\prime}} \otimes N_{X^{\prime}}\right|_{\mathscr{Q}_{0}}$ where $\left.N_{X^{\prime}}\right|_{\mathscr{Q}_{0}}$ is the normal bundle of $X^{\prime}$ in $\mathscr{Q}_{0}$. But since $X^{\prime}$ is a complete intersection, we have

$$
\left.N_{X^{\prime}}\right|_{\mathscr{Q}}=\left.N_{\mathscr{Q}_{1}}\right|_{X^{\prime}}
$$

where $\left.N_{\mathscr{Q}_{1}}\right|_{X^{\prime}}$ is the normal bundle of $\mathscr{Q}_{1}$ in $\mathbf{P}(\mathscr{V})$ restricted to $X^{\prime}$. Therefore

$$
\begin{aligned}
K_{X^{\prime}} & =\left.\left.K_{\mathscr{Q}_{0}}\right|_{X^{\prime}} \otimes N_{\mathscr{Q}_{1}}\right|_{X^{\prime}}=\left.\left(K_{\mathscr{Q}_{0}} \otimes N_{\mathscr{Q}_{1}}\right)\right|_{X^{\prime}}=\left.\left(K_{\mathbf{P}(\mathscr{V})} \otimes N_{\mathscr{Q}_{0}} \otimes N_{\mathscr{Q}_{1}}\right)\right|_{X^{\prime}} \\
& =\left.\left(\left.\left.\xi^{-5} \otimes \pi^{*}\left(K_{Y} \otimes \operatorname{det}(\mathscr{V})\right) \otimes\left[\mathscr{Q}_{0}\right]\right|_{\mathscr{Q}_{0}} \otimes\left[\mathscr{Q}_{1}\right]\right|_{\mathscr{Q}_{1}}\right)\right|_{X^{\prime}} \\
& =\left.\left(\xi^{-5} \otimes \pi^{*}\left(K_{Y} \otimes \operatorname{det}(\mathscr{V})\right) \otimes \xi^{2} \otimes \pi^{*}\left(\mathscr{M}_{0}\right) \otimes \pi^{*}\left(\mathscr{M}_{1}\right)\right)\right|_{X^{\prime}} .
\end{aligned}
$$

The last inequality holds because $X^{\prime} \cong \mathscr{Q}_{0} \cap \mathscr{Q}_{1}$.

Hence $K_{X^{\prime}}=\left.\left.\xi^{-1}\right|_{X^{\prime}} \otimes \pi^{*}\left(K_{Y} \otimes \operatorname{det}(\mathscr{V}) \otimes \mathscr{M}_{0} \otimes \mathscr{M}_{1}\right)\right|_{X^{\prime}}$.

Now since $K_{F}=\left.K_{X^{\prime}}\right|_{F}$ where $F$ is a general fibre of $\pi_{X^{\prime}}$ we see that $\left(K_{F}\right)^{-1}$ is very ample on $F$. Moreover $F$ is a complete intersection of two quadrics in the corresponding fibre, $\mathrm{P}^{4}$ of $\pi$. Hence $F$ must be a del Pezzo surface of degree 4.

(2.3.4) REMARK. Since $Y$ is smooth, it follows from the above proof that $K_{Y} \otimes \operatorname{det}(\mathscr{V}) \otimes \mathscr{M}_{0} \otimes \mathscr{M}_{1}=\mathscr{O}_{Y}$. We thus have a relationship between $\mathscr{M}_{0}$ and $\mathscr{M}_{1}$. Hence $X^{\prime}$ is completely determined if we know one of them.

(D) The case $r=7$ and $r=8$ (see (b) below).

(2.4.0) REMARK. Before proceeding we need to note the following facts:

(a) A smooth del Pezzo surface of degree 7 has exactly three exceptional curves, and exactly one of them meets each of the other two in a point of multiplicity one.

(b) A smooth del Pezzo surface of degree 8 has a unique exceptional curve. It is also possible for this surface to be isomorphic to $\mathbf{P}^{1} \times \mathbf{P}^{1}$, but we do not consider such surfaces in the following discussion.

(c) If $E$ denotes the exceptional curve in each of (a) and (b), and $F$ denotes the general fibre of $p$ as in (2.0), and $\left(X^{\prime}, L^{\prime}\right)$ as in (0.6.1), then by $(0.11)$ it follows that $L^{\prime} \cdot E=-K_{X^{\prime}} \cdot E=-K_{F} \cdot E$ (by adjunction and since $E$ is in $F, E \cdot F=0$ in $X^{\prime}$ ). But by adjunction $K_{F} \cdot E+E \cdot E=-2$, so that $K_{F} \cdot E=-1$. Hence $L^{\prime} \cdot E=1$.

(2.4.1) TheOREM. Let $p, X^{\prime}, L^{\prime}$ be as in (2.0). Assume moreover that $L$ is spanned. Then there exists a morphism to a threefold $X^{\prime \prime}$ such that 
the following diagram of morphisms commutes.

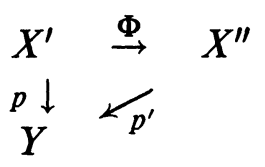

Moreover the general fibre of $p^{\prime}$ is $\mathbf{P}^{2}$.

Proof. Let $E, F$ be as in (2.4.0) (c), then we have

$$
0 \rightarrow N_{E / F} \rightarrow N_{E / X^{\prime}} \rightarrow N_{F / X^{\prime}, E} \rightarrow 0
$$

where $N_{A / B}$ is the normal bundle of $A$ in $B$, and $N_{A / B, C}$ is its restriction to $C$.

Since $E \cdot E=-1$ in $F$ and $N_{F / X}=\mathscr{O}_{F}$, we have

$$
0 \rightarrow \mathscr{O}_{E}(-1) \rightarrow N_{E / X^{\prime}} \rightarrow \mathscr{O}_{E} \rightarrow 0 .
$$

Hence

$$
N_{E / X^{\prime}} \cong \mathscr{O}_{E}(-1) \oplus \mathscr{O}_{E} .
$$

Let $\mathscr{H}$ denote the Hilbert scheme parametrizing smooth deformations of $E$ in $X^{\prime}$. Let $\mathscr{Z}$ denote its universal family. We have the following morphisms:

$$
\begin{gathered}
q: \mathscr{Z} \rightarrow X^{\prime} \\
r: \mathscr{Z} \rightarrow \mathscr{H} \quad \text { with } r \text { a flat morphism. }
\end{gathered}
$$

Let $\mathscr{D}=q(\mathscr{Z})$.

Claim. $\mathscr{D}$ is a $\mathbf{P}^{1}$-bundle over $Y$.

The general fibre of $r$ is $\mathbf{P}^{1}$, and since $L_{E}^{\prime} \cong \mathscr{O}_{E}(1)$ by (2.4.0) (c), and $r$ is flat, we have for any fibre $f$ of $r, \operatorname{deg}\left(L_{f}^{\prime}\right)=1$, and since $L_{f}^{\prime}$ is also ample and spanned by (1.6.2) $f \cong \mathbf{P}^{1}$, so that $\mathscr{Z}$ is a $\mathbf{P}^{1}$-bundle over $\mathscr{H}$. Hence $\mathscr{D}$ is a $\mathbf{P}^{1}$-bundle over $Y$. This proves the claim.

Since $N_{\mathscr{D} / X^{\prime}, E}=\mathscr{O}_{E}(-1), \mathscr{D}$ can be blown down smoothly to give a map $\Phi: X^{\prime} \rightarrow X^{\prime \prime}$, and this in turn gives us a morphism $p^{\prime}: X^{\prime \prime} \rightarrow Y$ whose general fibre is $\mathbf{P}^{2}$ in the case where $E$ is as in (2.4.0) (b), and in case $E$ is as in (2.4.0) (a), we are reduced to (2.4.0) (b), and this in turn gives us the desired morphism.

(2.4.2) Remark. Hence the cases $d=7$ and $d=8$ are reduced to the case $d=9$, and as we shall see, in this case $X^{\prime}$ is indeed a $\mathbf{P}^{2}$-bundle over $Y$.

(E) The case $d=9$. 
(2.5.1) Theorem. Let $p, X^{\prime}, S^{\prime}$ be as in (2.0). Assume moreover that $L$ is spanned by global sections, then $X^{\prime}$ is a $\mathbf{P}^{2}$-bundle over $Y$.

Proof. By $(0.11), K_{X^{\prime}}+L^{\prime}=p^{*}(\mathscr{M})$. Let $F\left(\cong \mathbf{P}^{2}\right)$ denote the general fibre of $p$. Let $C$ be an irreducible curve in $F$. Then $p(C)$ is a point. Hence by $[M, 3.6]$, there exists an extremal ray $R$, and a map $\eta=\operatorname{cont}_{R}$ such that the following diagram of morphisms commutes:

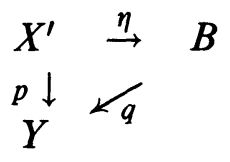

and $1 \leq \operatorname{dim} B \leq 3$.

Proof. We first try to prove the following

Claim. $\operatorname{dim} B=1$.

Proof (of claim). If $\operatorname{dim} B=3$, then by [M, 3.5], there exists $D$ such that:

(i) $D \cong \mathbf{P}^{2}$, and $\mathscr{O}_{D}(D)=\mathscr{O}_{\mathbf{P}^{2}}(-1)$

(ii) $D \cong \mathbf{P}^{2}$, and $\mathscr{O}_{D}(D)=\mathscr{O}_{\mathbf{P}^{2}}(-2)$

(iii) $D \cong \mathbf{P}^{1} \times \mathbf{P}^{1}$, and $\mathscr{O}_{D}(D)=\mathscr{O}(-1,-1)$

(iv) $D \cong$ to an irreducible quadric in $\mathbf{P}^{3}$, and $\mathscr{O}_{D}(D)=\left.\mathscr{O}_{\mathbf{P}^{3}}(-1)\right|_{D}$

(v) $D \cong \mathbf{P}^{-1}$-bundle over $\eta(D)$.

In (i), (ii), (iii) and (iv), $\eta(D)=$ point $=b$ (say), and since the above diagram commutes $D$ lies in $p^{-1}(y)$ for $y=q(b) \in Y$.

Case (a). $p^{-1}(y)$ is irreducible.

In cases (i), (ii), (iii) and (iv) since $D$ lies in $p^{-1}(y)$, we have $p^{-1}(y)=D$.

Hence $D$ is a fibre of $p$, and so $\mathscr{O}_{D}(D)=\mathscr{O}_{D}$ which contradicts (i), (ii), (iii) and (iv). We will consider cases (v) separately.

Case (b). $p^{-1}(y)$ is reducible, and $D$ is a component of $p^{-1}(y)$. We first note that $\left(p_{S^{\prime}}\right)^{-1}(y)=p^{-1}(y) \cap S^{\prime}$, which by (1.7) can be of type $\mathrm{I}_{b}, 1 \leq b \leq 9$, II, III, IV, and $\mathrm{I}_{b}^{*}, b=0,1$. We first note that in each of these types there is at least one component $C_{j}$ (say), with multiplicity one. Hence $S^{\prime}$ being ample in $X^{\prime}$, there is one component of $p^{-1}(y)=\bigcup_{i} F_{i}$ that is irreducible and reduced, say $F_{j}$. Also since $C_{j}^{2}=-2$ on $S^{\prime}$, by [So 5, 1.0.1 and 1.6], $p^{-1}(y)=\mathbf{P}^{2} \cup \mathbf{F}_{2}$, where $\mathbf{F}_{2}$ is the second Hirzebruch surface, on noting that the same proof carries through with the assumption on $\log \left(X^{\prime}, S^{\prime}\right)$ replaced by $\log \left(X^{\prime}, S^{\prime}\right)=1$. 
So $p^{-1}(y)=\mathbf{P}^{2} \cup \mathbf{F}_{2}$, and $\mathbf{P}^{2} \cap \mathbf{F}_{2}=C$, where $C$ is that unique curve on $\mathbf{F}_{2}$ such that $C^{2}=-2$.

Since $L^{\prime}$ is locally spanned $\mathbf{P}^{2}$ and $\mathbf{F}_{2}$ intersect transversely. Since $\left.\left(K_{X^{\prime}}+L^{\prime}\right)\right|_{\mathbf{P}^{2}}=\mathscr{O}_{\mathbf{P}^{2}}$, by adjunction and (i) and (ii), we have

$$
L_{\mathbf{P}^{2}}^{\prime} \cong \mathscr{O}_{\mathbf{P}^{2}}(e), \quad \text { where } e=1 \text { or } 2 .
$$

Similarly since $\left.\left(K_{X^{\prime}}+L^{\prime}\right)\right|_{\mathbf{F}_{2}} \cong \mathscr{O}_{\mathbf{F}_{2}}$ we have:

$$
L_{\mathbf{F}_{2}}^{\prime}-N_{\mathbf{F}_{2}}=\mathscr{O}_{\mathbf{F}_{2}}(2,4) \text {. }
$$

We also know that $\operatorname{deg} L_{\mathbf{P}^{2}}^{\prime}+\operatorname{deg} L_{\mathbf{F}_{2}}^{\prime}=9$. Since $\operatorname{deg} L_{\mathbf{P}^{2}}^{\prime}=1$ or 4 by (*), we therefore have $\operatorname{deg} L_{\mathbf{F}_{2}}^{\prime}=8$ or 5 , but since $\operatorname{deg} \mathbf{F}_{2}$ is even, perhaps $\operatorname{det} L_{\mathbf{F}_{2}}^{\prime}=8$ is possible. So letting $L_{\mathbf{F}_{2}}^{\prime}=(a, b)$, we have $a(b-a)=4$, so that $(a, b)=(1,5)$ or $(2,4)$. If $L_{\mathbf{F}_{2}}^{\prime}=(1,5)$, then by $(* *) N_{\mathbf{F}_{2}}=(-1,1)$. Now since $C=(1,0)$ on $\mathbf{F}_{2}$, we have $N_{\mathbf{F}_{2}} \cdot C=3$. On the other hand we also have

$$
N_{\mathbf{F}_{2} / X, C}=N_{C / \mathbf{P}^{2}}=\mathscr{O}_{\mathbf{P}^{2}}(1)
$$

since $\mathbf{P}^{2}$ and $\mathbf{F}_{2}$ intersect transversely in $C$.

Thus $N_{\mathbf{F}_{2}} \cdot C=1$ which is a contradiction. Also since $b>2 a$, $L_{\mathbf{F}_{2}}^{\prime}=(2,4)$ is impossible. Thus (i) and (ii) are impossible. This brings us to (iii).

Here $D\left(\cong \mathbf{P}^{1} \times \mathbf{P}^{1}\right)$ is a component of $p^{-1}(y)$. If $D_{1}$ is another component (there could be more) that intersects $D$ transversely, then by $(1.7) L^{\prime} \cdot D \cdot D_{1}=1$, or that $L_{D}^{\prime} \cdot\left[D_{1}\right]_{D}=1$, which is impossible since the intersection numbers on $\mathbf{P}^{1} \times \mathbf{P}^{1}$ are even. This brings us to (iv).

Here $D$ is an irreducible reduced singular quadric in $\mathbf{P}^{3}$. Since $\left.\left(K_{X^{\prime}}+L^{\prime}\right)\right|_{D}=\mathscr{O}_{D}$, by adjunction

$$
K_{D}+L_{D}^{\prime}=\mathscr{O}_{D}(D)=\left.\mathscr{O}_{\mathbf{P}^{3}}(-1)\right|_{D}
$$

Hence

$$
\operatorname{deg} K_{D}+\operatorname{deg} L_{D}^{\prime}=-2 .
$$

Since $L_{D}^{\prime}$ is ample $\operatorname{deg} L_{D}^{\prime} \geq 4$, so that $\operatorname{deg} K_{D} \leq-6$, which is impossible because $\operatorname{deg} K_{D}=8$. So it remains to disprove the possibility of $(\mathrm{v})$.

In (v) $\eta(D)$ is a curve and $D \cong \mathbf{P}^{1}$-bundle over $\eta(D)$. Here we have two possibilities:

$$
q(\eta(D))=p(D)=\left\{\begin{array}{l}
\text { a point } y \text { (say) } \\
\text { a curve }
\end{array}\right.
$$


(I) $p(D)=y$ then we have

Case (a) $D$ is irreducible, here $D=p^{-1}(y)$. If we let $f\left(\cong \mathbf{P}^{1}\right)$ denote the fibre of $\left.\eta\right|_{D}$, then $N_{D / X^{\prime}, f}=\mathscr{O}_{\mathbf{P}^{1}}$, which is not possible.

Case (b) $p^{-1}(y)$ is reducible and $D$ is a component of $p^{-1}(y)$. Here we see that $L_{D}^{\prime}$ is a smooth rational curve by (1.7), and is also ample in $D$. So $C \in\left|L_{D}^{\prime}\right|$ cannot be a fibre of $\left.\eta\right|_{D}$. Since $\eta(D)$ is a smooth curve, by Hurwitz formula $\left(\mathbf{P}^{1} \cong\right) C \cong \eta(D)$, and so $D \cong \mathbf{P}^{1} \times \mathbf{P}^{1}$. This is impossible, as we saw in case (iii) reducible case.

(II) $p(D)=$ curve, then $p(D)=Y$. Let $c$ denote a generic point of $Y$, then $p^{-1}(c)=\mathbf{P}^{2}$, and $\left(p_{D}\right)^{-1}(c)=\mathbf{P}^{2} \cap D=\left(\eta_{D}\right)^{-1}\left(q^{-1}(c)\right)=$ $\left(\eta_{D}\right)^{-1}(g)=\mathbf{P}^{1}$, where $g \in \eta(D)$. Hence $\mathbf{P}^{2} \cap D=\mathbf{P}^{1}$, so $N_{\mathbf{P}^{1} / \mathbf{P}^{2}}=$ $N_{D / X^{\prime}, \mathbf{P}^{1}}$. But then if $f$ is as in case (a), then $N_{D / X^{\prime}, f} \cong \mathscr{Q}_{\mathbf{P}^{1}}(-1)$. Hence $N_{\mathbf{P}^{1} / \mathbf{P}^{2}} \cong \mathscr{O}_{\mathbf{P}^{1}}(-1)$, which is impossible. Hence we conclude that $\operatorname{dim} B \leq 2$.

If $\operatorname{dim} B=2$, then for a generic $y \in Y, q^{-1}(y)$ is a smooth curve. Also $p^{-1}(y)=\mathbf{P}^{2}$. Hence by the commutativity of the diagram we find that $\eta$ restricted to $p^{-1}(y)$ gives a morphism onto a curve. But as is well known we cannot have a morphism from $\mathbf{P}^{2}$ onto a curve. Hence we must have $\operatorname{dim} B=1$. This proves the claim.

To finish the proof of the theorem; since $\operatorname{dim} B=1$, and the fibres of $p$ are connected. By the commutativity of the diagram $B \cong Y$. Hence by [M, 3.5.2], we conclude that $X^{\prime}$ is a $\mathbf{P}^{2}$-bundle over $Y$.

The following cases are a result of the observations and suggestions made by the referee:

(F) The cases $d=1$ and 8 .

In the case $d=1$, then we need the following proposition:

(2.6.0) Proposition. If $F$ is a smooth del Pezzo surface of degree 1 , then there exists a 2:1 morphism $\phi: F \rightarrow Q$, where $Q$ is a singular quadric in $\mathbf{P}^{3}$, branched along the vertex of $Q$ and a cubic curve that does not pass through the vertex of $Q$.

Proof. See [D-P-T p. 68-69].

(2.6.1) THEOREM. If the general fibre of $p$ is a del Pezzo surface of degree 1 , then there exists a morphism $\Phi: X^{\prime} \rightarrow p_{*}\left(-2 K_{X^{\prime}}\right)$, where $p_{*}\left(-2 K_{X^{\prime}}\right)$ is a quadric bundle over $Y$, and $\Phi$ is $2: 1$ and branched over the vertex and a cubic section over $Y$. 
Proof. The morphism in (2.6.0) is given by $\left|-2 K_{F}\right|$. Since the general fibre $F$ of $p$ is a del Pezzo surface of degree 1 , and $\left.\left[-2 K_{X^{\prime}}\right]\right|_{F} \cong$ $-2 K_{F}$ by adjunction; by Propositions (1.2) and (2.6.0) we are done.

Before mentioning the remaining cases we need the following:

(2.7) Definition (see [Se]). A field $k$ is said to be $C_{1}$, if for a homogeneous polynomial of degree $d \neq 0$ in $n$ variables with coefficients in $k$, whose only zero in $k^{n}$ is $(0, \ldots, 0)$, then $d \geq n$.

(2.7.1) Theorem. If $F$ is a del Pezzo surface of degree $d \geq 3$, defined over $a C_{1}$ field $k$ then it always has a $k$-rational point defined over it.

Proof. See [Ma; 4.1] and [S-D].

(2.7.2) REMARK. If $\mathbb{C}(Y)$ denotes the function field of $Y$, then $\mathbb{C}(Y)$ is $C_{1}$.

(2.7.3) Theorem. If the general fibres of $p$ are del Pezzo surfaces $F$, where $F \cong \mathbf{P}^{1} \times \mathbf{P}^{1}$, then there exists a birational morphism $\Psi: X^{\prime} \rightarrow X^{\prime \prime}$, where $X^{\prime \prime}$ is a $\mathbf{P}^{2}$-bundle over $Y$.

Proof. By (2.7.2), since the function field of $Y$ is $C_{1}$ there exists a section $s: Y \rightarrow X^{\prime}$. We blow-up $s(Y)$ in $X^{\prime}$. Let $\sigma$ denote this blowup map. This amounts to blowing up a point in the general fibre $F$ $\left(\cong \mathbf{P}^{1} \times \mathbf{P}^{1}\right)$ of $p$. Hence we are reduced to the case $d=7$, and so by (2.4.1) there exists a morphism, say $\zeta$ onto a $\mathbf{P}^{2}$-bundle over $Y$. Letting $\Psi=\zeta \circ \sigma$, we get the required birational map.

An Example. Let $C$ be an elliptic curve that is an $r$-sheeted branched covering of $\mathbf{P}^{1}$, where $1 \leq r \leq 8$. Embed this curve into $\mathbf{P}^{2}$ via the linear system $\left|3 P_{0}\right|$ (see [Ha 1] for details). We can embed this curve into $\mathbf{P}^{1} \times \mathbf{P}^{2}$ using the product of these two morphisms. Then $S \in\left|\pi_{1}^{*}\left(\mathscr{O}_{\mathbf{P}^{1}}(d)\right) \otimes \mathscr{O}_{\mathbf{P}^{2}}(3)\right|$ for $d$ sufficiently large positive number, gives us a very ample divisor in $\mathbf{P}^{1} \times \mathbf{P}^{2}$. If we blow-up this curve in $\mathbf{P}^{1} \times \mathbf{P}^{2}$, and if $X$ is the resulting threefold, and $b$ is the blow-up map, then $b^{*}([S])$, is again an ample line bundle over $X$, if $r \leq 7$ (see [Ha 1] Ch. iv, for details). If $r=8$, then it is spanned by global sections. Hence we have the following commutative diagram:

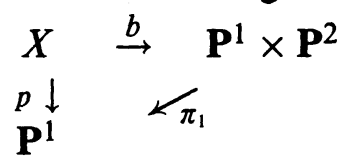


The general fibre of $p$ is a del Pezzo surface of degree $(9-r)$.

REMARK. One has to worry about the general position of the $r$ points in the fibre of the branched covering of $\mathbf{P}^{1}$ in the embedding of the curve into $\mathbf{P}^{1} \times \mathbf{P}^{2}$, because the blowing up of the curve might not result in a del Pezzo surface for the general fibre.

Acknowledgments. The author thanks his thesis advisor A. Sommese for all the help and encouragement received, and also thanks to the referee for the many suggestions included in section $(F)$ and for the elegant proof of (0.7). The author would also like to thank L. Fania for the many useful ideas in the case $d=9$; I. Dolgachev and B. Crauder for all the helpful discussions during the course of this paper.

\section{REFERENCES}

[B\&H] E. Bombieri and D. Husemoller, Classification and embeddings of surfaces, Proc. Symp. Pure Math., 29, ed by R. Hartshorne, (1975), 329-420.

[D-P-T] Demazure-Pinkham-Tessier, Séminaire sur les Singularités des Surfaces, Springer Lecture Notes in Mathematics \#777.

[F-So] M. L. Fania and A. Sommese, On the minimality of hyperplane sections of threefolds, Contribution to several complex variables (Proceedings of the conference in Honor of Professor Wilhelm Stoll) in Vieweg (1985).

[Ha 1] R. Hartshorne, algebraic Geometry, Springer-Verlag, 1977, GTM \#52.

[Ha 2] _ Ample Subvarieites of Algebraic Varieties, Springer Lecture Notes Series \#156.

[I1] S. Iitaka, On logarithmic Kodaira dimension of algebraic varieties, Complex Analysis and Algebraic Geometry, ed. by W. L. Baily, Jr. T. Shioda, 1977, 175-189, Iwanami Shoten.

[12] _ Algebraic Geometry, Springer-Verlag, 1982, GTM \#76.

[Ko] K. Kodaira, On compact analytic surfaces: II, Ann. of Math., 77 (1963), 563.

[Ma] Jurii I. Manin, Rational surfaces over perfect fields, Publ. Math. IHES, 30, 99-113.

[M] S. Mori, Threefolds whose canonical bundles are not numerically effective, Ann. of Math., 116 (1982), 133-176.

[Se] J. P. Serre, Local Fields, GTM, Springer Verlag.

[So 1] A. Sommese, On manifolds that cannot be ample divisors, Math. Ann., 221 (1976), 55-72.

[So 2] _ Adjunction theoretic structure of projective varieties, Springer Lecture notes \#1115, Proceedings of the Complex Geometry Conference at Gottingen in May 1985.

[So 3] _ Hyperplane sections of projective surfaces: I-the adjunction mapping, Duke Math. J., 46 (1979), 377-401.

[So 4] - Hyperplane Sections, Proceedings, Chicago Circle Conference, Springer Lecture notes \#862, 1980. 
[So 5] - On the configurations of -2 rational curves on hyperplane sections of projective threefolds, classifications of algebraic and analytic manifolds, Prog. Math., 39 (1980), 465-497.

[So 6] The birational theory of hyperplane sections of projective threefolds, (preprint).

[S-D] H. P. F. Swinnerton-Dyer, Rational points on del Pezzo surfaces of degree 5, Wolters-Noordhoff, Proc. of 5th Nordic Summer School, Oslo, Norway. Ed. F. Oort.

[VdV] Van de Ven, On the 2-connectedness of very ample divisors on a surface, Duke Math. J., 46 (1979), 403-407.

Received December 8, 1986.

UNiversity of Michigan, Flint

FLINT, MI 48502-2186 


\section{PACIFIC JOURNAL OF MATHEMATICS EDITORS}

V. S. VARADARAJAN (Managing Editor) University of California Los Angeles, CA 90024

HeRbert Clemens

University of Utah

Salt Lake City, UT 84112

R. FINN

Stanford University

Stanford, CA 94305
ROBION KIRBY

University of California

Berkeley, CA 94720

C. C. MOORE

University of California

Berkeley, CA 94720

HAROLD STARK

University of California, San Diego

La Jolla, CA 92093

\section{ASSOCIATE EDITORS}
R. ARENS
E. F. BECKENBACH
B. H. NEUMANN
F. Wolf
K. YOSHIDA (1906-1982)

\section{SUPPORTING INSTITUTIONS}

UNIVERSITY OF ARIZONA UNIVERSITY OF OREGON

UNIVERSITY OF BRITISH COLUMBIA UNIVERSITY OF SOUTHERN CALIFORNIA

CALIFORNIA INSTITUTE OF TECHNOLOGY UNIVERSITY OF CALIFORNIA MONTANA STATE UNIVERSITY STANFORD UNIVERSITY

UNIVERSITY OF NEVADA, RENO UNIVERSITY OF HAWAII NEW MEXICO STATE UNIVERSITY UNIVERSITY OF TOKYO UNIVERSITY OF UTAH OREGON STATE UNIVERSITY WASHINGTON STATE UNIVERSITY UNIVERSITY OF WASHINGTON 


\section{Pacific Journal of Mathematics}

Vol. 134, No. $1 \quad$ May, 1988

Marco Abate, Annular bundles . ............................

Ralph Cohen, Wen Hsiung Lin and Mark Mahowald, The Adams

spectral sequence of the real projective spaces

Harry Joseph D'Souza, Threefolds whose hyperplane sections are elliptic surfaces .........................................57

Theodore Gerard Faticoni, Localization in finite-dimensional FPF rings . . . 79

Daniel Hitt, Invariant subspaces of $\mathscr{H}^{2}$ of an annulus ................ 101

Ellen Kirkman and James J. Kuzmanovich, On the global dimension of fibre products

Angel Rafael Larotonda and Ignacio Zalduendo, Homogeneous spectral sets and local-global methods in Banach algebras

Halsey Lawrence Royden, Jr., Invariant subspaces of $\mathscr{H}^{p}$ for multiply

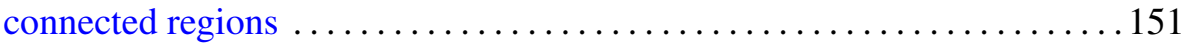

Jane Sangwine-Yager, A Bonnesen-style inradius inequality in 3-space . . . . 173

Stefano Trapani, Holomorphically convex compact sets and cohomology

Thomas Vogel, Uniqueness for certain surfaces of prescribed mean

curvature 